\title{
Responses of Soil Properties and Bacterial Community to the Application of Sulfur Fertilizers in Black and Sandy Soils
}

\author{
Siqi Dong, Shouran Liu, Shuai Cui, Xue Zhou*, Qiang Gao** \\ Key Laboratory of Sustainable Utilization of Soil Resources in The Commodity Grain Bases of Jilin Province, \\ College of Resource and Environmental Sciences, Jilin Agricultural University, Changchun, China,
}

Received: 20 February 2021

Accepted: 17 June 2021

\begin{abstract}
Sulfur (S) is an essential nutrient element for crops and plays an important role in regulating and controlling the growth and development of plants. However, there are few studies on the effects of inorganic $\mathrm{S}$ fertilizer on soil bacterial community structure and the relationship between these communities and soil properties in the main corn producing areas of Jilin Province, China. This study evaluated the effects of applying different levels of ammonium sulfate fertilizers (S0, S30, S90, S150) on soil properties and bacterial communities. The results revealed that $\mathrm{S}$ fertilizer treatment increased the available sulfur (AS) content in soil and reduced soil pH, soil organic matter (OM) as compared with S0 treatment. S fertilizer treatment increased the bacterial richness of black soil (Chaol) and reduced the bacterial richness of sandy soil. In addition, the application of S fertilizer significantly changed the soil bacterial community structure and it was revealed that $\mathrm{S}$ fertilizer have different effect on black and sandy soil bacterial communities. Partial Least Squares Discriminant Analysis (PLS-DA) indicated that the $\mathrm{S}$ treatment changed the structure of bacterial community and Redundancy analysis (RDA) showed that soil $\mathrm{pH}$ and $\mathrm{AS}$ were major variables affecting the structure of soil bacterial community.
\end{abstract}

Keywords: sulfur fertilizer, soil type, bacterial community structure, high-throughput sequencing

\section{Introduction}

Sulfur (S) is the fourth nutrient element after $\mathrm{N}, \mathrm{P}$ and $\mathrm{K}$ [1]. It is an essential nutrient element for crops and plays an important role in regulating and controlling the growth and development of plants. Plants have a higher demand for $\mathrm{S}$ as they do for the other

*e-mail: zhouxue_jlau@163.com

**e-mail: gyt9962@126.com macro nutrient elements [2]. $\mathrm{S}$ in plants is a structural component of certain coenzymes and prosthetic groups. It is mainly involved in protein synthesis and also participates in various physiological and biochemical reactions [3]. Plants use $90 \%$ of $\mathrm{S}$ in plants is used to synthesize sulfur-containing amino acids to promote chlorophyll synthesis and root growth [4]. Plants need S in their whole life cycle, and their demand are internally adjusted according to environmental conditions and plant growth stages [5]. Hence, S plays an important role in the farmland ecosystem. 
The main source of soil $\mathrm{S}$ is the deposition of air pollutants [6]. Recently studies revealed that, the amount of $\mathrm{S}$ emissions in the atmosphere has been greatly reduced and the insufficient application of sulfur-containing fertilizers and the reduction in the use of sulfur-containing pesticides have caused the lack of biologically effective $\mathrm{S}$ in soils [7]. Crops rely on $\mathrm{S}$ in the soil to synthesize proteins and some essential cofactors. Deficiency of S not only affects crop yield and quality, but also reduces its utilization of $\mathrm{N}, \mathrm{P}$ and $\mathrm{K}$ $[8,9] . \mathrm{S}$ in the soil exists in both organic and inorganic forms and about $95 \%$ of the $\mathrm{S}$ is in the organic state. However, the organic S usually go through different transformation processes such as activation, fixation, mineralization, oxidation and reduction before it can be absorbed and utilized by plants [10].

The interrelationship between microorganisms, soil and crops is the main driving force of the agricultural ecosystem [11]. Soil microorganisms are the key to maintaining soil properties, and play an important regulatory role in nutrient cycling, decomposition and conversion of nutrients, and improvement of soil structure. Studies have shown that microorganisms can convert organic $\mathrm{S}$ into inorganic $\mathrm{S}$ for plant absorption and utilization [12]. This process is mediated by both fungi and bacteria in the soil. During $\mathrm{S}$ oxidation, fungi are less efficient than bacteria [13]. The dominant bacterial groups involved in the $\mathrm{S}$ cycle are different, such as autotrophic oxidizing bacteria and is oxygen oxidizing bacteria [14]. A large number of microorganisms use inorganic sulfide as energy for metabolism covering a broad range of taxa consisting of Alphaproteobacterial, Betaproteobacteria and Gammaproteobacteria; Actinobacteria; and Firmicutes [15]. Soil microorganism respond differently to various fertilization conditions, which may be attributed to different soil types and the amount of fertilizer [16]. In different soil types, $\mathrm{S}$ in the fertilizer will be physically or biologically fixed, which affects the utilization rate of $\mathrm{S}$. The input of inorganic fertilizers can directly provide nutrients. However, it may affect the soil microbial groups involved in the material cycle. Previous studies reported on the application of nitrogen and phosphate fertilizers [17], furthermore, applying S fertilizer will reveal the same results.

Fertilization has an impact on the physical and chemical properties of the soil can change the microbial community structure. In order to understand the impact of different amounts of $\mathrm{S}$ fertilizer on the bacterial community structure of different soil types (black soil and sandy soil) two different fertilization experiments were set under the same climatic conditions, for both
S. The purpose of this study was to understand the response of bacteria and plants to different $\mathrm{S}$ fertilizer application rates on two different soil types from maize production areas in Northeast China and to evaluate the effects of different amounts of sulfate fertilizer on the abundance, composition, and structure of soil bacteria while corn absorbs sulfate. A series of changes in soil physical and chemical properties of soil is caused by the conversion of organic $\mathrm{S}$ into inorganic $\mathrm{S}$ mediated by soil bacteria guide the rational application of $\mathrm{S}$ fertilizer. Although the soil microbial flora plays an important role in the process of plant S supply and soil $\mathrm{S}$ cycle, there is less data available in literature on the effect of inorganic S fertilizer on the structure of soil microbial community.

\section{Materials and Methods}

\section{Field Sites and Experiment Description}

The experimental sites were located in Sankeshu $\left(43^{\circ} 20^{\prime} \mathrm{N}, 124^{\circ} 00^{\prime} \mathrm{E}\right)$ and Fujia $\left(43^{\circ} 21^{\prime} \mathrm{N}, 124^{\circ} 05^{\prime} \mathrm{E}\right)$, Siping, Jilin Province, China. The two experimental sites had the same climatic conditions (temperate monsoon climate), but different soil types. According to the World Reference Base (WRB) soil classification system, soils collected from Sankeshu and Fujia were divided into Chernozems and Arenosols, respectively [18]. The two sites characterized by an annual rainfall of $717.4 \mathrm{~nm}$ and an average annual temperature of $6.6^{\circ} \mathrm{C}$. The basic soil properties were measured at the beginning of the field test (Table 1). Research plots were set up to study the effects of S fertilization (ammonium sulfate) application rates on maize yields and soil microorganisms. The $\mathrm{S}$ fertilization rates were $\mathrm{S} 0$ (0 kg S ha-1), S30 (30 kg S ha $\left.{ }^{-1}\right)$, S90 (90 kg S ha-1) and $\mathrm{S} 150\left(150 \mathrm{~kg} \mathrm{~S} \mathrm{ha}{ }^{-1}\right) ; \mathrm{S}$ fertilization rate were applied across 24 plots at the two sites. The application rates, fertilizer types and plot sizes are summarized in (Table 2). One-time fertilization was applied to each treatment. Maize was planted in May at a planting density of 65,000 ha-1, and harvested in October.

\section{Soil Sampling and Analyses}

Soil samples were collected in September 2018, and the sampling tools were sterilized prior to use. In each plot 3 maize were selected randomly. The roots were shaken gently to remove any loosely adhering soil and then the rhizosphere soil that was tightly adhering to the roots was carefully detached using a brush and

Table 1. Basic soil physical and chemical properties.

\begin{tabular}{|c|c|c|c|c|c|c|c|}
\hline Location & Soil Types & $\mathrm{pH}$ & $\mathrm{OM}(\mathrm{g} / \mathrm{kg})$ & $\mathrm{AN}(\mathrm{mg} / \mathrm{kg})$ & $\mathrm{AP}(\mathrm{mg} / \mathrm{kg})$ & $\mathrm{AK}(\mathrm{mg} / \mathrm{kg})$ & $\mathrm{AS}(\mathrm{mg} / \mathrm{kg})$ \\
\hline Sankeshu & Black & 6.63 & 23.2 & 106.57 & 38.37 & 191.29 & 13.24 \\
\hline Fujiajie & Sandy & 5.77 & 16.2 & 61.71 & 24.96 & 160.92 & 11.36 \\
\hline
\end{tabular}


Table 2. Pure nutrient input.

\begin{tabular}{|c|c|c|c|c|}
\hline \multicolumn{5}{|c|}{ Pure nutrient input $(\mathrm{kg} / \mathrm{ha})$} \\
\hline Treatment & $\mathrm{S}$ & $\mathrm{N}$ & $\mathrm{P}_{2} \mathrm{O}_{5}$ & $\mathrm{~K}_{2} \mathrm{O}$ \\
\hline $\mathrm{S} 0$ & 0 & 210 & 90 & 90 \\
\hline $\mathrm{S} 30$ & 30 & 210 & 90 & 90 \\
\hline $\mathrm{S} 90$ & 90 & 210 & 90 & 90 \\
\hline $\mathrm{S} 150$ & 150 & 210 & 90 & 90 \\
\hline
\end{tabular}

mixed after sieving $(2 \mathrm{~mm})$. Each of the eight treatments (two soil type $\times$ four $\mathrm{S}$ fertilization rates) consisted of 3 independent replicates resulting in a total of 24 samples. Each soil sample was divided into two equal subsamples: one subsample was used for soil property analysis, and the other subsample was stored at $-80^{\circ} \mathrm{C}$ for subsequent DNA extraction.

Soil $\mathrm{pH}$, soil organic $\mathrm{C}$ content, available nitrogen (AN), available phosphorus (AP) and available potassium (AK) were measured according to methods described by [19]. The AS content is extracted with $\mathrm{KH}_{2} \mathrm{PO}_{4}$, measured with Inductive Coupled Plasma Emission Spectrometer (ICP) [20].

\section{Illumina MiSeq High-Throughput Sequencing and Data Analysis}

Soil DNA was extracted using the Fast DNA SPIN extraction kits (MP Biomedicals, Santa Ana, CA, USA), following the manufacturer's instructions, and stored at $-20^{\circ} \mathrm{C}$ prior to further analysis.

PCR amplification of the bacterial $16 \mathrm{~S}$ rRNA genes (V3-V4 regions) region was performed using the forward primer $338 \mathrm{~F}$ (5'-ACTCCTACGGGAGGCAGCA-3') and the reverse primer 806R (5'-GGACTACHVGGGTWTCTAAT-3'). PCR amplicons were purified with Agencourt AMPure Beads (Beckman Coulter, Indianapolis, IN) and quantified using the PicoGreen dsDNA Assay Kit (Invitrogen, Carlsbad, CA, USA). After the individual quantification step, amplicons were pooled in equal amounts, and pair-end $2 \times 300$ bp sequencing was performed using the Illlumina MiSeq platform with MiSeq Reagent Kit v3 at Shanghai Personal Biotechnology Co., Ltd (Shanghai, China).

The Quantitative Insights Into Microbial Ecology (QIIME, v1.8.0) pipeline was employed to process the sequencing data, as previously described [21]. Briefly, raw sequencing reads with exact matches to the barcodes were assigned to respective samples and identified as valid sequences. After chimera detection, the remaining high-quality sequences were clustered into operational taxonomic units (OTUs) at $97 \%$ sequence identity by UCLUST [22]. A representative sequence was selected from each OTU using default parameters.. The QIIME (v1.8.0) software was used to analyze alpha indexes (including Shannon, Simpson,
Chaol and ACE indexes).

\section{Statistical Analysis}

Differences in soil properties across samples were determined by ANOVA in IBM SPSS (version 25.0, Chicago, IL, USA). Spearman's correlation coefficient was used to investigate the potential correlation between diversity and soil AS using SPSS16.0. The "plsda" function in R (3.6.0) package "mixOmics" was used to represent Partial Least Squares Discriminant Analysis (PLS-DA) [23]. R was used to complete a heat map for to the analysis of the relative abundance of bacterial communities. Redundancy analysis (RDA) was generated in Canoco 5.

\section{Results}

\section{Soil Physicochemical Properties}

The $\mathrm{S}$ application rate increased as the soil $\mathrm{pH}$ value decreased (Fig. 1). The experimental results of the two soil types were consistent, with a decrease of 0.05-0.32 units compared with S0, and the differences were significant. The AS gradually increased with the input of S fertilizer, and reached the highest in S150 treatment. According to ANOVA analysis, the amount of $\mathrm{S}$ fertilizers showed correlation with soil $\mathrm{pH}$ and AS. The amount of $\mathrm{S}$ fertilizers was significantly correlated with $\mathrm{OM}$ and other measured soil properties (AN, AK, AP) showed no significant changes.

\section{Characteristics of the Soil Microbiome Community Structure}

In total, we obtained 1050279 valid sequences were obtained from 24 soil samples, and 34159-51243 sequences were obtained per sample $($ mean $=4376$ ). After excluding OUTs whose abundance were less than $0.001 \%, 296352$ OTUs were retained.

To study whether the correlation between fertilizers and bacterial diversity indicators changes after being applied to the soil, the alpha diversity index in this article is analyzed by 4 indicators. Chaol and ACE index are used to evaluate the total number of OTUs contained in the sample. The larger the value, the higher the abundance of the flora. In black soil, the application of S fertilizer reduced the species abundance as compared to S0, however, the Chaol index of S150 treatment increased significantly. In sandy soil, $\mathrm{S}$ fertilizer application increased as the species abundance significantly decreased. Shannon and Simpson indexes were used to estimate the diversity of microbial communities. The larger the value, the higher the diversity. The analysis revealed, that in black soil, the bacterial diversity of soil without $\mathrm{S}$ fertilizer was higher than that with fertilization, and the results were opposite in sandy soil. The Simpson index showed no change. 
With the increase of $\mathrm{S}$ application in black soil and sandy soil, the Chaol index showed a downward trend, indicating that fertilization would cause the microbial species in sandy soil and black soil to gradually become unitary. The Shannon index in the two soils declined slightly, but not significantly (Table 3 ).

\section{Soil Bacterial Community Composition}

The dominant bacteria phylum with relative abundance were greater than $1 \%$ in the two soil types were similar. The top five phyla which relative abundances accounted for about $90.9 \%$ of the total sequence were: Actinobacteria (38.7\%), Proteobacteria (30.7\%), Acidobacteria (7.8\%), Gemmatimonadetes (7.2\%), Chloroflexi (6.5\%). Actinobacteria has a higher abundance in sandy soil; the Proteobacteria and Gemmatimonadetes abundance are higher in black soil. The abundance of other bacterial phylum did not change significantly, but there were differences between treatments. The abundance of Proteobacteria and Actinobacteria in the two soils were significantly

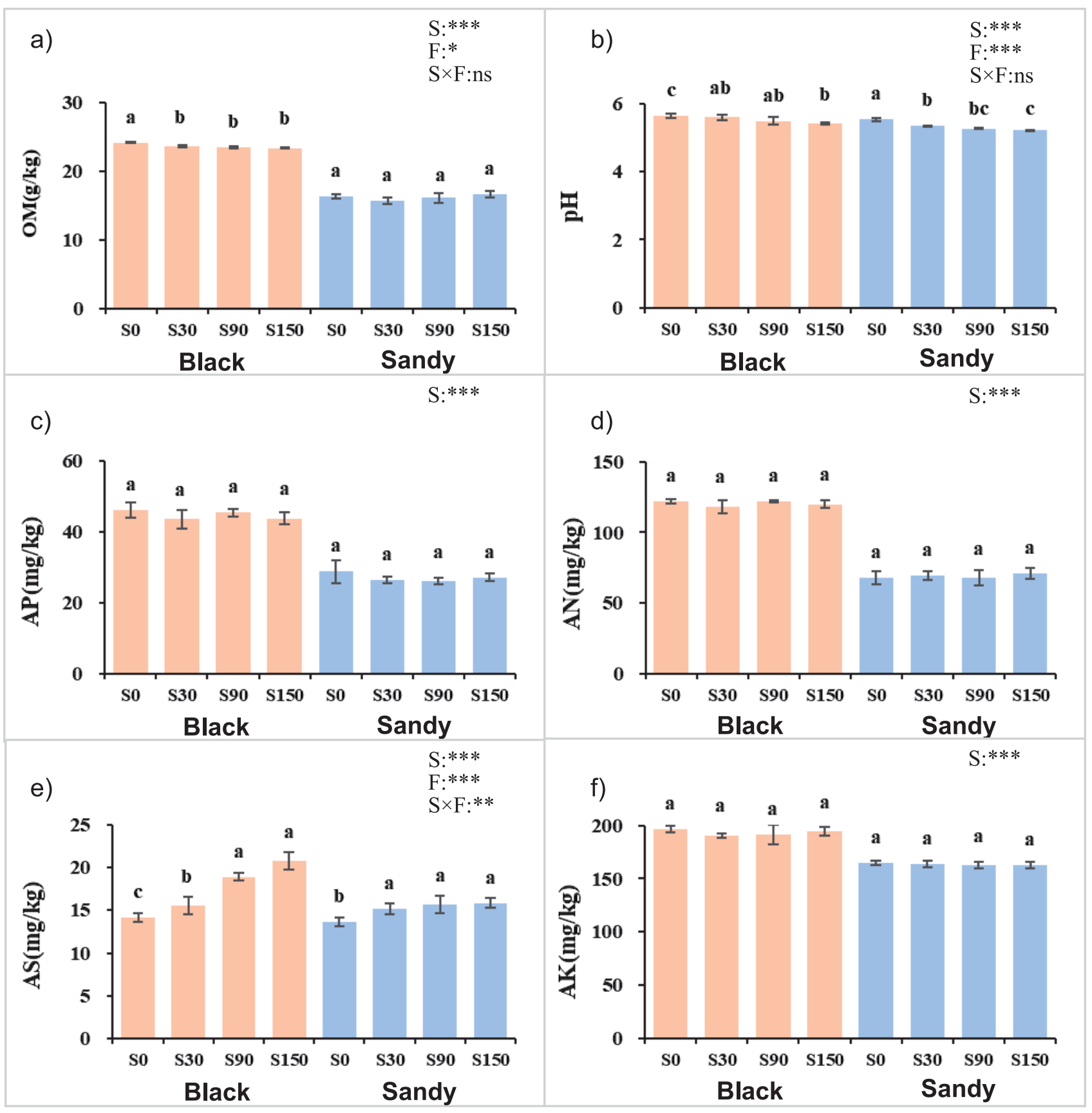

Fig. 1. The physicochemical properties of the soil vary with the amount of S fertilizer applied. In order to measure the overall impact of soil type $(\mathrm{S})$, fertilizers $(\mathrm{F})$ and their interaction $(\mathrm{S} \times \mathrm{F})$ on soil variables, two-way ANOVAs was carried out. Different lowercase letters indicate significant differences between treatments estimated by one-way ANOVA and Duncan's multiple-range test and asterisks denote significant differences at $p<0.05, p<0.01$ and $p<0.001$ probability levels ( $* * *$ and $* * *)$. 
Table 3. Diversity indices of soil microbial community under application of S fertilizers in two types of soils.

\begin{tabular}{|c|c|c|c|c|c|}
\hline Soil Types & Treatment & Chao1 index & ACE index & Shannon index & Simpson index \\
\hline Black & S0 & $3353 \pm 551.3 \mathrm{ab}$ & $3393 \pm 608.0 \mathrm{ab}$ & $10.35 \pm 0.05 \mathrm{a}$ & $0.9977 \pm 0.0001 \mathrm{ab}$ \\
\hline & S30 & $2953 \pm 124.8 \mathrm{~b}$ & $3049 \pm 187.2 \mathrm{~b}$ & $10.22 \pm 0.08 \mathrm{~b}$ & $0.9974 \pm 0.0004 \mathrm{~b}$ \\
\hline & S90 & $3282 \pm 496.7 \mathrm{ab}$ & $3292 \pm 471.1 \mathrm{ab}$ & $10.33 \pm 0.07 \mathrm{ab}$ & $0.9979 \pm 0.0002 \mathrm{a}$ \\
\hline & S150 & $3938 \pm 117.0 \mathrm{a}$ & $3939 \pm 116.9 \mathrm{a}$ & $10.27 \pm 0.04 \mathrm{ab}$ & $0.9978 \pm 0.0001 \mathrm{ab}$ \\
\hline & S0 & $3105 \pm 267.3 \mathrm{a}$ & $3232 \pm 285.9 \mathrm{a}$ & $9.93 \pm 0.05 \mathrm{a}$ & $0.9974 \pm 0.0001 \mathrm{a}$ \\
\hline & S30 & $2778 \pm 154.2 \mathrm{ab}$ & $2844 \pm 168.7 \mathrm{ab}$ & $9.94 \pm 0.09 \mathrm{a}$ & $0.9973 \pm 0.0002 \mathrm{a}$ \\
\hline & S90 & $2597 \pm 110.5 \mathrm{~b}$ & $2617 \pm 138.9 \mathrm{~b}$ & $10.05 \pm 0.04 \mathrm{a}$ & $0.9976 \pm 0.0001 \mathrm{a}$ \\
\hline & S150 & $2644 \pm 336.9 \mathrm{~b}$ & $2699 \pm 363.4 \mathrm{~b}$ & $9.97 \pm 0.34 \mathrm{a}$ & $0.9974 \pm 0.0005 \mathrm{a}$ \\
\hline
\end{tabular}

Different lowercase letters indicated the significant difference at the $5 \%$ level.

affected by the interaction of soil type and the $\mathrm{S}$ fertilizer. The soil type changed the relative abundance of Bacteroidetes, Gemmatimonadetes, Proteobacteria, Actinobacteria and Acidobacteria; and the amount of $\mathrm{S}$ fertilizer changed the relative abundance of Bacteroidetes and Patescibacteria (Fig. 2).

Further taxonomical classification revealed that Sphingomonas $\quad(2.5 \%-11.4 \%)$, Jatrophihabitans (1.4\%-7.3\%), Gemmatimonas (1.1\%-7.7\%), Bradyrhizobium (1.8\%-2.7\%) and Nocardioides $(1.5 \%-2.5 \%)$ were the dominant genera across all treatments. The addition of $\mathrm{S}$ fertilizer significantly affected the relative abundance of 6 different genera, including Haliangium $(P=0.009)$, Bryobacter $(P=0.022)$, Chujaibacter $(P=0.049)$, Arthrobacter $(P=0.012)$, Singulisphaera $(P=0.006)$ and Mycobacterium $(P=0.035)$ (Fig. 3).

\section{Bacterial Community Distribution}

In order to determine whether different $\mathrm{S}$ application rates will cause changes in soil bacterial communities in both black and sandy soils, PLS-DA was used to analyze changes in soil bacterial community composition. The community structure of the soil bacteria shifted significantly as a result of $\mathrm{S}$ fertilization, and clear distinction among the different $\mathrm{S}$ treatments and soil types were observed (Fig. 4) while in sandy soils, bacterial community composition showed more clear separation between different $\mathrm{S}$ application rates.

Comparative analysis of taxa with significant differences in phylum and genus levels (Table 4). Under the influence of the same fertilization gradient, the different taxa of black soil were lower than that of sandy soil. However, in the two soils S90

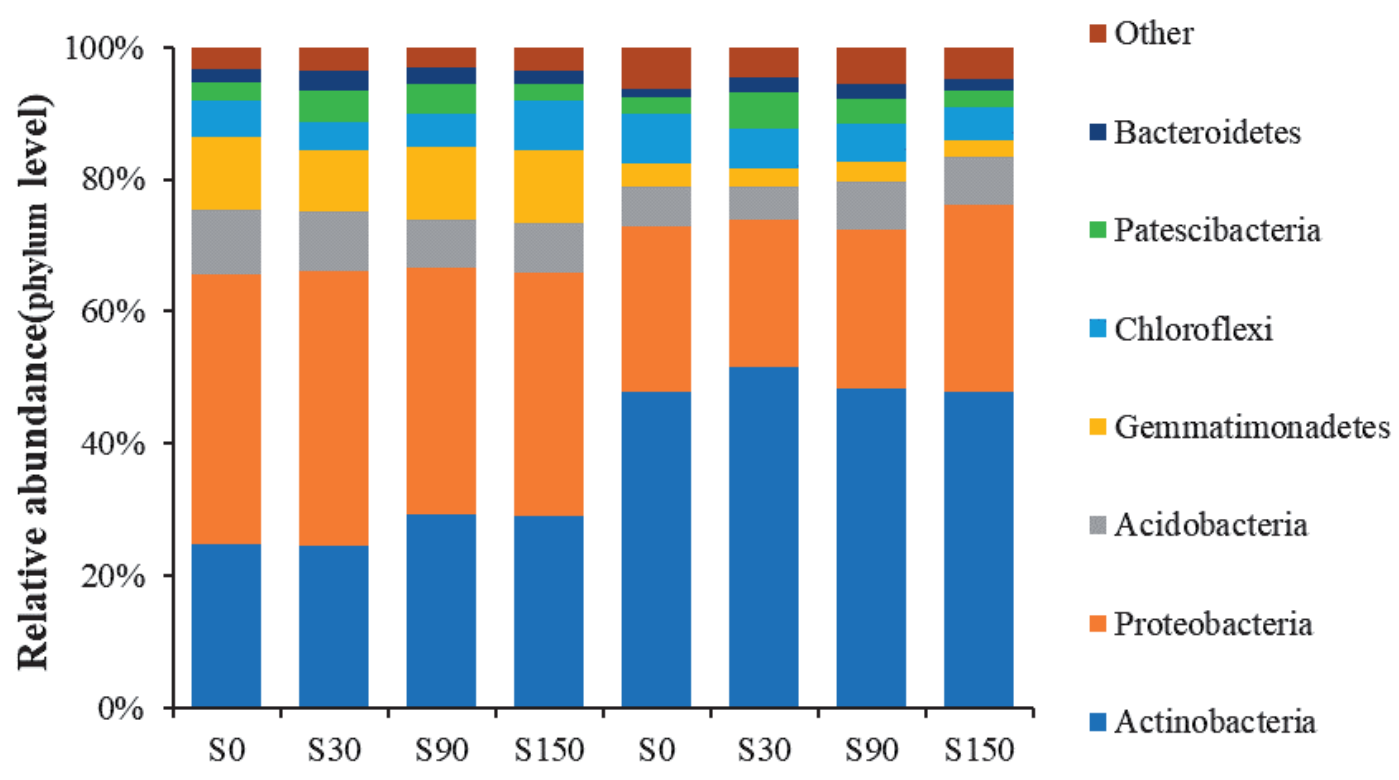

Fig. 2. The relative abundance levels of the dominant phylum under different treatments of the two soil types. In order to measure the overall impact of soil type (S), fertilizers (F) and their interaction $(\mathrm{S} \times \mathrm{F})$ on phylum level bacterial community composition, two-way ANOVAs was carried out. Asterisks denote significant differences at $p<0.05, p<0.01$ and $p<0.001$ probability levels $\left(*, * *\right.$ and $\left.{ }^{* * *}\right)$. 
treatment, although the phylum level has three types of bacteria different, not completely the same (black soil: Bacteroidetes, Acidobacteria and Patescibacteria; sandy soil: Firmicutes, Acidobacteria and Nitrospirae), other treatments have also been the same argument (Table S1). The above results show that the bacterial communities in different soil types respond differently to $S$ fertilizer input. Therefore, the results of the partial least squares method have also been verified, and the input of different $\mathrm{S}$ fertilizers has a greater impact on the bacterial community structure in aeolian sandy soil.

\section{Environmental and Bacterial Community Distribution}

Redundancy Analysis (RDA) was used to analyze variations in bacterial community structure and its associations with environmental variables. In

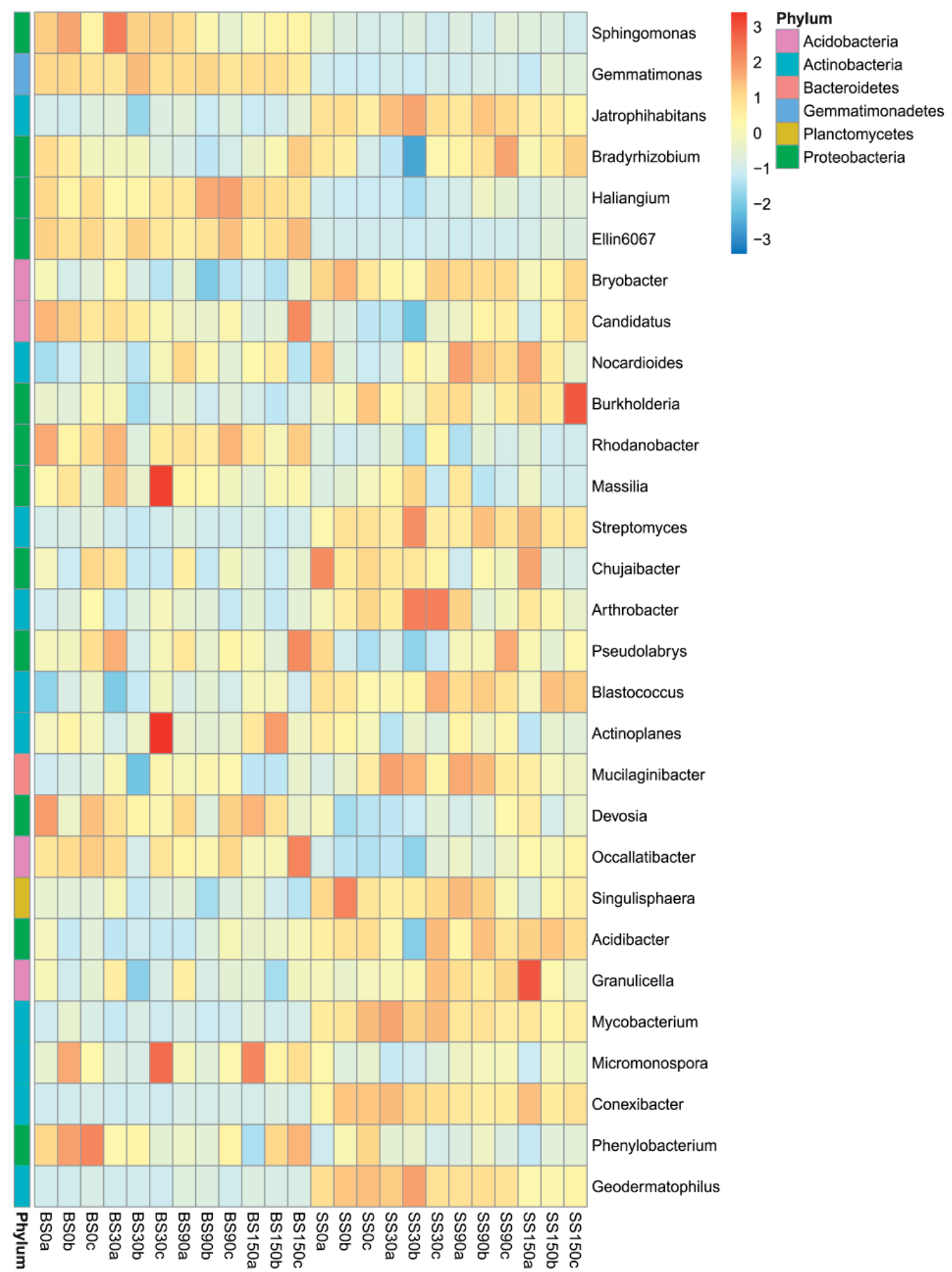

Fig. 3. The heatmap shows the bacterial taxonomic variations (genus level with abundance greater than $0.1 \%$ ) over S fertilization and soil types., the asterisk $\left(^{*}\right)$ indicates the $p<0.05, p<0.01$ and $p<0.001\left({ }^{*}, * *\right.$ and $\left.{ }^{* * *}\right)$ according to the Pearson correlation between their relative abundances and AS application rates. 
Table 4. The number of taxa (phylum level and genus level) with significant differences between different treatments, group means comparison between two treatments.

\begin{tabular}{|c|c|c|c|c|c|}
\hline Group & Phylum & Genus & Group & Phylum & Genus \\
\hline BS30-BS0 & 0 & 21 & SS30-SS0 & 3 & 30 \\
\hline BS90-BS0 & 3 & 18 & SS90-SS0 & 3 & 43 \\
\hline BS150-BS0 & 1 & 19 & SS150-SS0 & 3 & 10 \\
\hline BS0-SS0 & 11 & 164 & BS90-SS90 & 9 & 143 \\
\hline BS30-SS30 & 10 & 144 & BS150-SS150 & & 133 \\
\hline
\end{tabular}

sandy soils (Fig. 5a), RDA1 revealed $16.68 \%$ of total variation, and this component negatively correlated with environmental parameters, such as $\mathrm{pH}, \mathrm{OM}$ and $\mathrm{AK}$. RDA1 positively correlates with AS, AP and AN. 9.93\% of total bacterial variation was revealed by RDA2. Both $\mathrm{OM}$ and $\mathrm{pH}$ positively correlated with the RDA2 component, and others negatively correlated with the RDA2. In black soils (Fig. 5b), RDA1 showed 15.44\% of total variation, and this component negatively correlated with environmental parameters, such as AP and AS. RDA1 positively correlates with AN, AK, $\mathrm{pH}$ and OM. RDA2 revealed $9.53 \%$ of total bacterial variation. Both $\mathrm{AS}$ and $\mathrm{AN}$ positively correlated with the RDA2 component, and others negatively correlated with the RDA2.

\section{Discussion}

The applied $\mathrm{S}$ fertilizer in the soil was stored as a short-term $\mathrm{S}$ reservoir through adsorption and fixation, and can undergo a series of migration and transformation in the soil, directly or indirectly affecting the properties of the soil. Studies have shown that the application of $\mathrm{S}$ fertilizer can reduce the $\mathrm{pH}$ value of weakly alkaline soils, and as the input of $\mathrm{S}$ fertilizer increases, the $\mathrm{pH}$ decreases more significantly [24]. Different amounts of desulfurized gypsum in salinealkali land were applied and revealed that desulfurized gypsum can effectively reduce the $\mathrm{pH}$ of saline-alkali soil [25]. This was mainly because $\left(\mathrm{NH}_{4}\right)_{2} \mathrm{SO}_{4}$ is an acid fertilizer. After applying $\left(\mathrm{NH}_{4}\right)_{2} \mathrm{SO}_{4}$, it was shown

PLS-DA

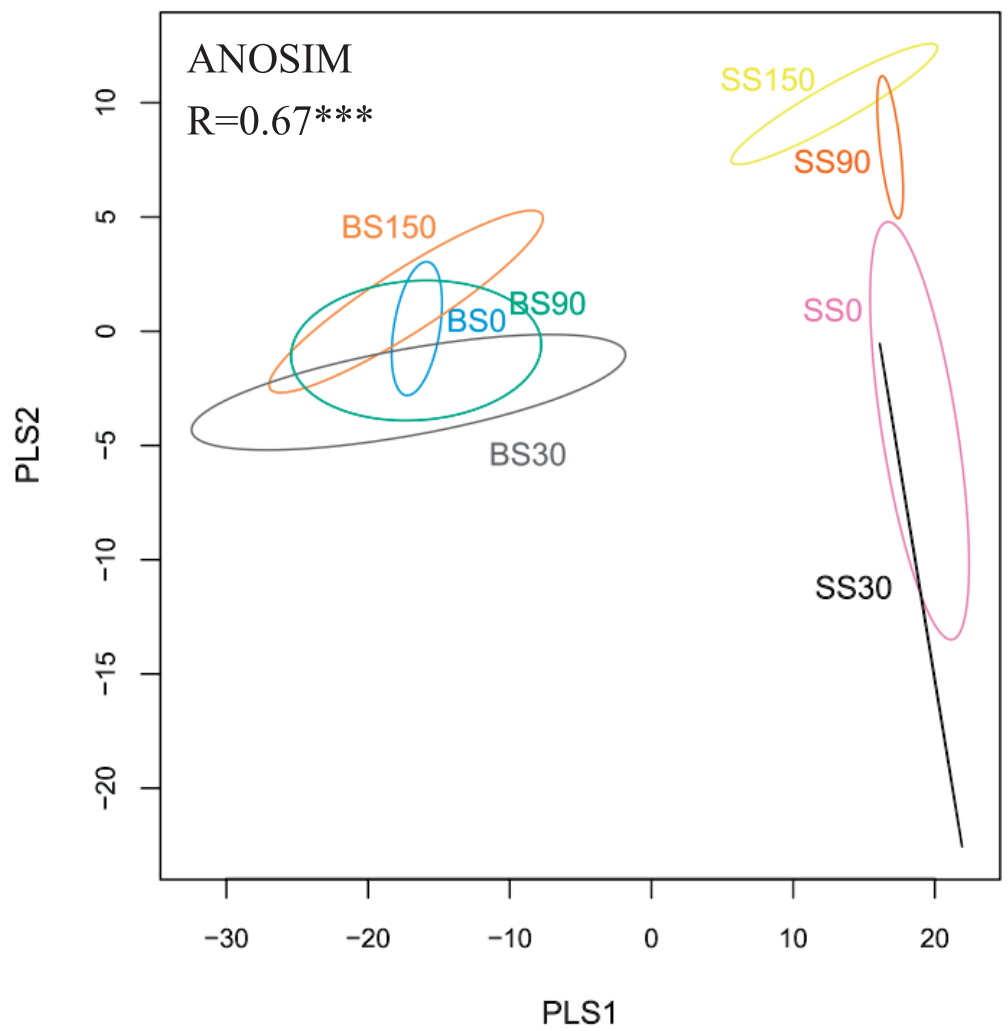

Fig. 4. Partial Least Squares Discriminant Analysis (PLS-DA) of different soil types and S fertilizer rates on soil bacterial communities. 


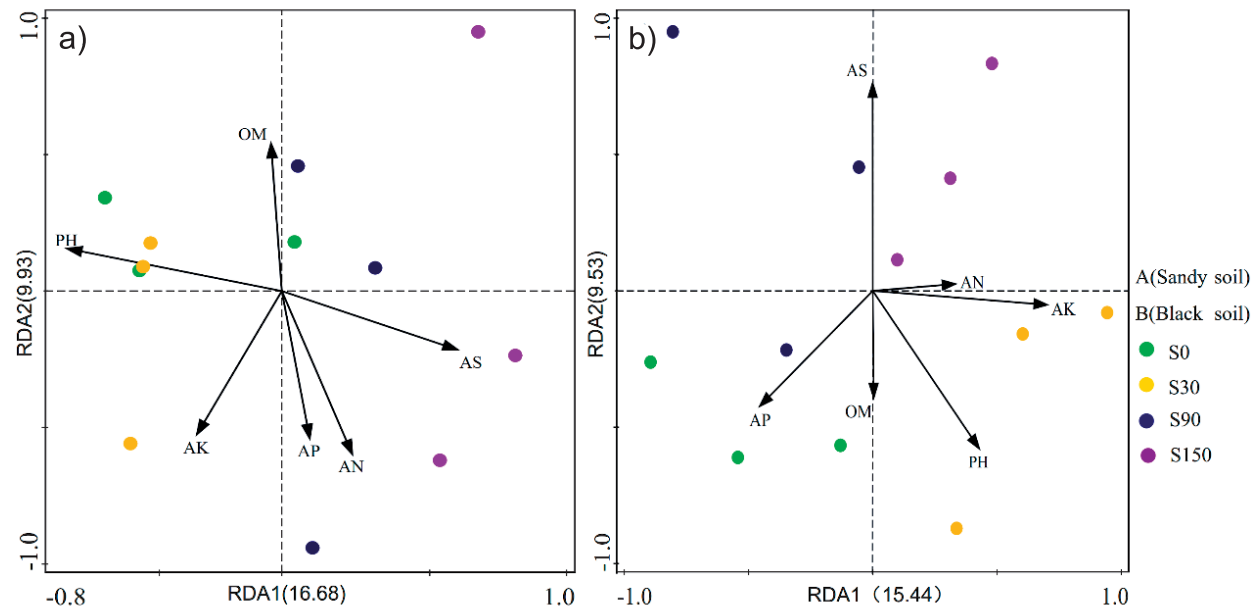

Fig. 5. Redundancy analysis (RDA) revealed the influence of soil properties on the structure of soil bacterial community, A(Sandy soil), B(Black soil). The green, yellow, blue, and purple circles represent different S application rates (S0, S30, S90, S150), Soil properties OM represent sorganic matter and AS represents available sulfur.

that plants absorbed $\mathrm{NH}_{4}^{+}$instead of $\mathrm{SO}_{4}^{2-}$, and released a large amount of $\mathrm{H}^{+}$during the absorption of ion exchange, which makes the soil acidify and lower the pH. Du's study in 2020 showed that with the increase in the amount of $\mathrm{S}$ fertilizer application, the soil available $\mathrm{S}$ content showed a significant increase trend [24]. The application of $\mathrm{S}$ fertilizer to the paddy field revealed that the concentration of thiosulfate and sulfide in potted plants with $\mathrm{CaSO}_{4}$ were slightly higher than that in both control and potted plants with $\mathrm{CaCO}_{3}$ fertilizer [26]. In this study, the application of S fertilizer reduced the $\mathrm{pH}$ of black soil and sandy soil, and the AS content gradually increased as the application of $\mathrm{S}$ fertilizer increases (Fig. 1). Previous studies have shown that the oxidation of soil sulfur was mainly carried out with the participation of soil microorganisms. The addition of sulfur fertilizer mainly affects the AS content by affecting the relative abundance of sulfate reducing bacteria [27].

There have been many researches which reported on the effects of fertilizers on soil bacterial richness and diversity [28]. However, there were few reports on the effects of sulfur-containing fertilizers on soil bacterial richness and diversity. We found that there was no significant difference in soil diversity index between Chaol and Shannon in the rhizosphere soil under different sulfur fertilizer treatments (Table 3). And the results of Tang showed that there were no significant differences in Ace, Chaol, Shannon and other diversity indexes of rhizosphere under different $\mathrm{S}$ treatments, which were consistent with our results [27]. However, previous studies revealed that $\mathrm{N}$ and $\mathrm{P}$ fertilizers could affect the soil bacterial diversity. Li et al.'s research showed that soil bacterial richness and diversity were significantly reduced with the increase of nitrogen appplication rate $[28,29]$. Chen's study showed that long-term phosphate fertilizer input on paddy soil increased bacterial abundance and diversity [30].

Due to the input of $\mathrm{S}$ fertilizer, the soil bacterial community structure showed a change, and this revealed that the effect of $\mathrm{S}$ fertilizer in black and sandy soil bacterial community were different (Fig. 2). The results of this study were consistent with the results of previous studies which obtained Actinobacteria, Proteobacteria, Acidobacteria, Gemmatimonadetes, Bacteroidetes, Chloroflexi and Patescibacteria as the dominant bacteria phyla, and their abundance accounts for about $90 \%$ of the entire phylum level [31]. Proteobacteria is a common dominant phylum in agricultural soils. This phylum contains nitrogen-fixing bacteria and bacteria related to the sulfate cycle [32]. The abundance of each bacteria is different in different types of soil samples, for example, the abundance of Actinobacteria and Proteobacteria in the two soils in this study (Fig. 4). There were many reported on the effects of soil physical and chemical factors on the changes of soil microbial communities [33]. Yu's research showed that $\mathrm{N}$ fertilization significantly affected soil physicochemical properties (such as $\mathrm{pH}$, organic $\mathrm{C}$ and $\mathrm{NO}_{3}^{-}$) and bacterial community structure in clay, alluvial and sandy soils; the response of the three soil bacterial community structures to $\mathrm{N}$ fertilization were different [19]. The RDA of this research explored the main factors affecting the soil bacterial communities, and the results showed that the dominate factors were $\mathrm{pH}$ and AS. The results obtained in this study were consistent with Cheng's research, which studied the influence of phosphorus fertilization patterns on the bacterial community in upland farmland, and revealed that $\mathrm{pH}, \mathrm{OM}$ and AP were important factors affecting the composition and diversity of the bacterial community [34]. Tang's research showed that AS content in rice 
was the most important factor affecting the composition of microbial community when applying $\mathrm{S}$ fertilizer [27].

At present, there are few studies on the effects of sulfate fertilization on soil microbial flora. In the future, the scope of research will be expanded to further explore the microbiological mechanisms that dominate the soil S cycle.

\section{Conclusions}

In general, the application of $\mathrm{S}$ fertilizer can significantly change the physical and chemical properties of the black and sandy soils. The application of $\mathrm{S}$ fertilizer changed the structure of soil bacterial community, and the effect of $\mathrm{S}$ fertilizer in black and sandy soil bacterial community were different. The diversity of bacteria in different soil types also affected by $\mathrm{S}$ fertilizer. Fertilization caused changes in the physical and chemical properties of the soil, which affected the bacterial community and would further change their potential function of the $\mathrm{S}$ cycle.

\section{Acknowledgments}

This work was supported by the National Natural Science Foundation (U19A2035), the Natural Science Foundation of Jilin Province, China (20190201274JC), and the Science and Technology Project of the $13^{\text {th }}$ FiveYear Plan of Jilin Provincial Department of Education (JJKH20190908KJ).

\section{Conflict of Interest}

The authors declare no conflict of interest.

\section{References}

1. MICHAEL A., KERTESZ., PASCAL MIRLEAUY. The role of soil microbes in plant sulphur nutrition. Journal of Experimental Botany. 55, 1939, 2004.

2. TAKAHASHI H., KOPRIVA S., GIORDANO M., SAITO K., HELL R. Sulfur assimilation in photosynthetic organisms: molecular functions and regulations of transporters and assimilatory enzymes. Annual Review of Plant Biology. 62, 157, 2011.

3. XIA F.F., SU Y., WEI X.M., HE Y.H., WU Z.C., GHULAM A., HE R. Diversity and activity of sulphur-oxidizing bacteria and sulphate-reducing bacteria in landfill cover soils. Lett Appl Microbiol. 59, 26, 2014.

4. ZHAO F.J., SALMON S.E., WITHERS PJA., EVANS EJ., MONAGHAN JM., SHEWRY PR., MCGRATH SP. Responses of breadmaking quality to sulphur in three wheat varieties. Journal of the Science of Food and Agriculture. 79, 1865, 1999.

5. BAO P., LI G.X., SUN G.X., XU Y.Y., ANDREW A.M., ZHU Y.G. The role of sulfate-reducing prokaryotes in the coupling of element biogeochemical cycling. Science of the Total Environment. 613, 398, 2018.

6. MCGRATH S.P., ZHAO F.J., BLAKE KALFF M.M.A. History and outlook for sulphur fertilizers in Europe, Proceedings 497 of the International Fertilizer Society, Cambridge, UK, 5-27, 2002 [In English].

7. CARCIOCHI W.D., WYNGAARD N., DIVITO G.A., REUSSI CALVO N.I., CABRERA M.L., ECHEVERRÍA H.E. Diagnosis of sulfur availability for corn based on soil analysis. Biology and Fertility of Soils, 52, 917, 2016.

8. SUTRADHAR A.K., KAISER D.E., FERNÁNDEZ F.G. Does total nitrogen/sulfur ratio predict nitrogen or sulfur requirement for corn. Soil Science Society of America Journal, 81, 564, 2017.

9. ABD ELKADER., MONA G. Effect of sulfur application and foliar spraying with zinc and boron on yield, yield components, and seed quality of peanut. Research Journal of Agriculture and Biological Sciences, 9 (4), 127, 2013.

10. ZHAO C., DEGRYSE F., GUPTA VVSR., MCLAUGHLIN M.J. Elemental sulfur oxidation in Australian cropping soils. Soil Science Society of America Journal, 79, 89, 2015.

11. SINSABAUGH R.L., BELNAP J., RUDGERS J., KUSKE C.R., MARTINEZ N. Soil microbial responses to nitrogen addition in arid ecosystems. Frontiers in Microbiology, 6, 819, 2015.

12. GAHAN J., SCHMALENBERGER A. The role of bacteria and mycorrhiza in plant sulfur supply. Frontiers in Plant Science, 5, 72, 2014.

13. SCHMALENBERGER A., NOLL M. Bacterial communities in grassland turfs respond to sulphonate addition while fungal communities remain largely unchanged. European Journal of Soil Biology, 61, 12, 2014.

14. ZHAO C., VADAKATTU V.S.R GUPTA., FIEN., DEGRYSE., MIKE J MCLAUGHLIN. Abundance and diversity of sulphur-oxidising bacteria and their role in oxidizing elemental sulphur in cropping soils. Biology and Fertility of Soils, 53, 159, 2016.

15. KERTESZ M.A., FROSSARD E. Soil Microbiology Ecology and Biochemistry, Academic Press, Boston, 471503, 2015 [In English].

16. LING N., SUN Y.M., MA J.H., GUO J.J., ZHU P., PENG C., YU G.G., RAN W., GUO S.W., SHEN Q.R. Response of the bacterial diversity and soil enzyme activity in particlesize fractions of mollisol after different fertilization in a long-term experiment. Biology and Fertility of Soil, 50, 901, 2014.

17. IKOYI I., FOWLER A., SCHMALENBERGER A. One-time phosphate fertilizer application to grassland columns modifies the soil microbiota and limits its role in ecosystem services. Science of The Total Environment, 630, 849, 2018.

18. ZHANG H.J., DING W.X., YU H.G., HE X.H. Linking organic carbon accumulation to microbial community dynamics in a sandy loam soil: result of 20 years compost and inorganic fertilizers repeated application experiment. Biology and Fertility of Soils, 51, 137, 2014.

19. YU H.L., GAO Q., SHAO Z.Q., YING A., SUN Y., LIU J., MAO W., ZHANG B. Decreasing nitrogen fertilizer input had little effect on microbial communities in three types of soils. PLoS ONE, 11, 0151622, 2016.

20. ANDCRSON G., LEFROY R., CHINOIM N., BLAIG R. Sulphur testing. Sulphur in Agriculture, 16, 6, 1992.

21. CAPORASO J.G., KUCZYNSKI J., STOMBAUGH J., BITTINGER K., BUSHMAN F.D., COSTELLO E.K., FIERER N., PENA A.G., GOODRICH J.K., GORDON 
J.I. QIIME allows analysis of high-throughput community sequencing data. Nature Methods, 7, 335, 2010.

22. EDGAR R.C. Search and clustering orders of magnitude faster than BLAST. Bioinformatics, 26 (19), 2460, 2010.

23. CHEN Y.F., YANG F.L., LU H.F., WANG B.H., CHEN Y.B., LEI D.J., WANG Y.Z., ZHU B.L., LI L.J. Characterization of Fecal Microbial Communities in Patients with Liver Cirrhosis. Hepatology, 54 (2), 562, 2011.

24. DU X.P., LIU G.P., PU P., LU J.Y., LI W.J., XU X.Y. Effects of $\mathrm{SO}_{4}{ }^{2-}-\mathrm{S}$ and $\mathrm{S}_{0}-\mathrm{S}$ on the sultur nutrition in Pakchoi. Journal of Nuclear Agricultural Sciences, 34 (3), 0635, 2020.

25. SKWIERAWSKA M., ZAWARTKA L., ZAWADSKI B. The effect of different rates and forms of sulphur applied on changes of soil agrochemical properties. Plant Soil and Environment, 54 (4), 171, 2008.

26. SACHIKO MASUDA., BAO Z.H., TAKASHI OKUBO., KAZUHIRO SASAKI., SEISHI IKEDA., SHINODA., MIZUE ANDA., RYUJI KONDO., YUMI MORI., KIW AMU MINA MISAW. Sulfur fertilization changes the community structure of rice root and soil associated bacteria. Microbes and Environment, 31 (1), 70, 2016.

27. TANG X.J., LI L.Y., WU C., MUHAMMED IMRAN KHAN., MARIA MANZOOR., ZOU L.N., SHI J.Y. The response of arsenic bioavailability and microbial community in paddy soil with the application of sulfur fertilizers. Environmental Pollution, 264, 1, 2020.

28. LI Y., CHENG N., LIU Y.H., DU W., HE P. Soil microbial community composition closely associates with specific enzyme activities and soil carbon chemistry in a long- term nitrogen fertilized grassland. Science of the Total Environment, 654, 264, 2019.

29. CAI S.M., WANG J.J., LV W.J., XU S.X., ZHU H.T. Nitrogen fertilization alters the effects of earthworms on soil physicochemical properties and bacterial community structure. Applied Soil Ecology, 150, 103478, 2020.

30. CHEN X.D., JIANG N., LEO M. CONDRONC., KARI E DUNFIELDD, CHEN Z.H., WANG J.K., CHEN L.J. Impact of long-term phosphorus fertilizer inputs on bacterial phoD gene community in a maize field, Northeast China. Science of the Total Environment, 669, 1011, 2019.

31. YANG Q.W, ZHONG J.H., XIA Q.M., ZHANG S.L., LI X., PAN X.Y., ZHAO R.P., WANG R.W.,YANG W.M., ZHOU P., SHANG G., HU F.Y., YANG C.D., WANG W. Soil microbial mechanisms promoting ultrahigh rice yield. Soil Biology and Biochemistry, 143, 107741, 2020.

32. DAQUIADO A.R., KUPPUSAMY S., KIM S.Y., KIM J.H., YOON Y.E., KIM P.J., OH S.H., KWAK Y.S. Pyrosequencing analysis of bacterial community diversity in long-term fertilized paddy field soil. Applied Soil Ecology, 108, 84, 2016.

33. ZHONG Y.Q.W., YAN W.M., WAN R.W., WANG W., SHANGGUAN Z.P. Decreased occurrence of carbon cycle functions in microbial communities along with long-term secondary succession. Soil Biology and Biochemistry, 123, 207, 2018

34. CHENG H.Y., YUAN M.S., DUAN Q.Y., SUN R.X., SHENY.F., YU Q. Influence of phosphorus fertilization patterns on the bacterial community in upland farmland. Industrial Crops Products, 155, 112761, 2020.

\section{Supplementary Material}

Table S1. Response of bacterial communities to S fertilizer input.

\begin{tabular}{|c|c|}
\hline \multicolumn{2}{|r|}{ Group (Phylum) } \\
\hline BS30-BS0 & \\
\hline BS90-BS0 & Bacteroidetes, Acidobacteria, Patescibacteria \\
\hline $\mathrm{BS} 150-\mathrm{BS} 0$ & Armatimonadetes \\
\hline SS30-SS0 & Firmicutes, Chloroflexi, Nitrospirae \\
\hline SS90-SS0 & Firmicutes, Acidobacteria, Nitrospirae \\
\hline SS150-SS0 & Acidobacteria, Firmicutes, Planctomycetes \\
\hline BS0-SS0 & $\begin{array}{c}\text { Firmicutes, WPS-2, Gemmatimonadetes, Actinobacteria, Verrucomicrobia, Proteobacteria, Elusimicrobia, } \\
\text { Nitrospirae, Acidobacteria, Chloroflexi, Planctomycetes }\end{array}$ \\
\hline BS30-SS30 & $\begin{array}{l}\text { Firmicutes, Actinobacteria,, Proteobacteria,, WPS-2, Nitrospirae, Gemmatimonadetes,, Elusimicrobia,, } \\
\text { Fibrobacteres,, Verrucomicrobia,, Planctomycetes }\end{array}$ \\
\hline BS90-SS90 & $\begin{array}{l}\text { Firmicutes, Actinobacteria, Proteobacteria, Elusimicrobia, Gemmatimonadetes, WPS-2, Planctomycetes, } \\
\text { Bacteroidetes, BRC1, Patescibacteria }\end{array}$ \\
\hline BS150-SS150 & $\begin{array}{c}\text { Actinobacteria, , WPS-2, Elusimicrobia, Proteobacteria, Gemmatimonadetes, Nitrospirae, Firmicutes, } \\
\text { Verrucomicrobia, Rokubacteria }\end{array}$ \\
\hline
\end{tabular}


Table S1. Continued.

\begin{tabular}{|c|c|}
\hline \multicolumn{2}{|r|}{ Group (geuns) } \\
\hline BS30-BS0 & $\begin{array}{c}\text { Belnapia, Frankia, Bosea, Dongia, ADurb.Bin063-1, Oxalicibacterium, Actinomadura, Enterobacter, Angustibacter, } \\
\text { Curtobacterium, Ohtaekwangia, metagenome, Catellatospora, Microbacterium, Segetibacter,Phenylobacterium, } \\
\text { Candidatus_Adlerbacteria_bacterium_GW2011_GWC1_50_9, Curvibacter, Variovorax, Escherichia-Shigella, } \\
\text { Piscinibacter, }\end{array}$ \\
\hline BS90-BS0 & $\begin{array}{c}\text { Candidatus_Fritschea, Edaphobacter, metagenome, Mucilaginibacter, Frateuria, Sphingobium, Solirubrobacter, } \\
\text { Thermus, Noviherbaspirillum, Pantoea, Candidatus_Solibacter, Bradyrhizobium, Phenylobacterium, Nocardioides, } \\
\text { Rhodopseudomonas, Afipia, Actinoplanes, Conexibact, }\end{array}$ \\
\hline BS150-BS0 & $\begin{array}{c}\text { Belnapia, Ideonella, Microbacterium, Pantoea, Sphingobium, Pelomonas, Duganella, Nordella, Janibacter, } \\
\text { Escherichia-Shigella, Curtobacterium, Rhodopseudomonas, Kaistia, Ochrobactrum, Afipia, Bauldia, metagenome, } \\
\text { Dethiobacter, Herbaspirillum }\end{array}$ \\
\hline SS30-SS0 & $\begin{array}{c}\text { Kineosporia, Tumebacillus, Qipengyuania, Humibacter, Leifsonia, Bacillus, Clostridium_sensu_stricto_1, } \\
\text { Psychroglaciecola, Christensenellaceae_R-7_group, Pseudarthrobacter, Turicibacter, Sporosarcina, Agromyces, } \\
\text { Acidiphilium, Aestuariimicrobium, Georgenia, Pseudoclavibacter, Actinoplanes, Flavobacterium, MND1, } \\
\text { Rugosimonospora, Castellaniella, Nitrospira, Microbacterium, Arcticibacter, Phaselicystis, Motilibacter, } \\
\text { Terrabacter, Angustibacter, Curtobacterium }\end{array}$ \\
\hline SS90-SS0 & $\begin{array}{l}\text { Asanoa, Bacillus, Tumebacillus, Kineosporia, Candidatus_Alysiosphaera, Modestobacter, Subgroup_10, } \\
\text { Granulicella, Craurococcus, Methylovirgula, Lacunisphaera, Lysobacter, Psychroglaciecola, Nakamurella, } \\
\text { Crossiella, Rubrobacter, Agromyces, Terriglobus, Sporosarcina, Motilibacter, Ralstonia, Rudaibacter, Candidatus_ } \\
\text { Solibacter, Microlunatus, Labrys, Pseudonocardia, Actinospica, Antricoccus, Occallatibacter, Nitrospira, } \\
\text { Qipengyuania, Bauldia, Acidothermus, Ramlibacter, Mucilaginibacter, Castellaniella, Legionella, Nitrosospira, } \\
\text { Nitrolancea, Haliangium, Afipia, Gemmatimonas, Sphaerisporangium }\end{array}$ \\
\hline SS150-SS0 & $\begin{array}{c}\text { Nevskia, Tumebacillus, Lysinimonas, Bacillus, Qipengyuania, Occallatibacter, Nakamurella, Craurococcus, Labrys, } \\
\text { Geodermatophilus, Sphaerisporangium, Aquisphaera, Castellaniella, Agromyces, Methylovirgula, Duganella, } \\
\text { Methylibium, Nitrosospira, Kribbella, Lapillicoccus, Bryocella, Rudaibacter, Acidovorax, Angustibacter, mle1-7, } \\
\text { Microlunatus, Parafrigoribacterium, Reyranella, Nitrolancea, Mesorhizobium, Actinospica, bacterium_WWH38, } \\
\text { Enterobacter, Motilibacter, Paludisphaera, Lysinibacillus, Amycolatopsis, Vulgatibacter, Naasia, Actinoplanes, } \\
\text { Acidibacter, Rudaeicoccus, Haliangium, Legionella, Lysobacter, Actinomycetospora, Sporosarcina, Kineosporia, } \\
\text { Xenophilus, Pseudoclavibacter, Acidisphaera, Solirubrobacter, Acidothermus, Allorhizobium-Neorhizobium- } \\
\text { Pararhizobium-Rhizobium }\end{array}$ \\
\hline BS0-SS0 & 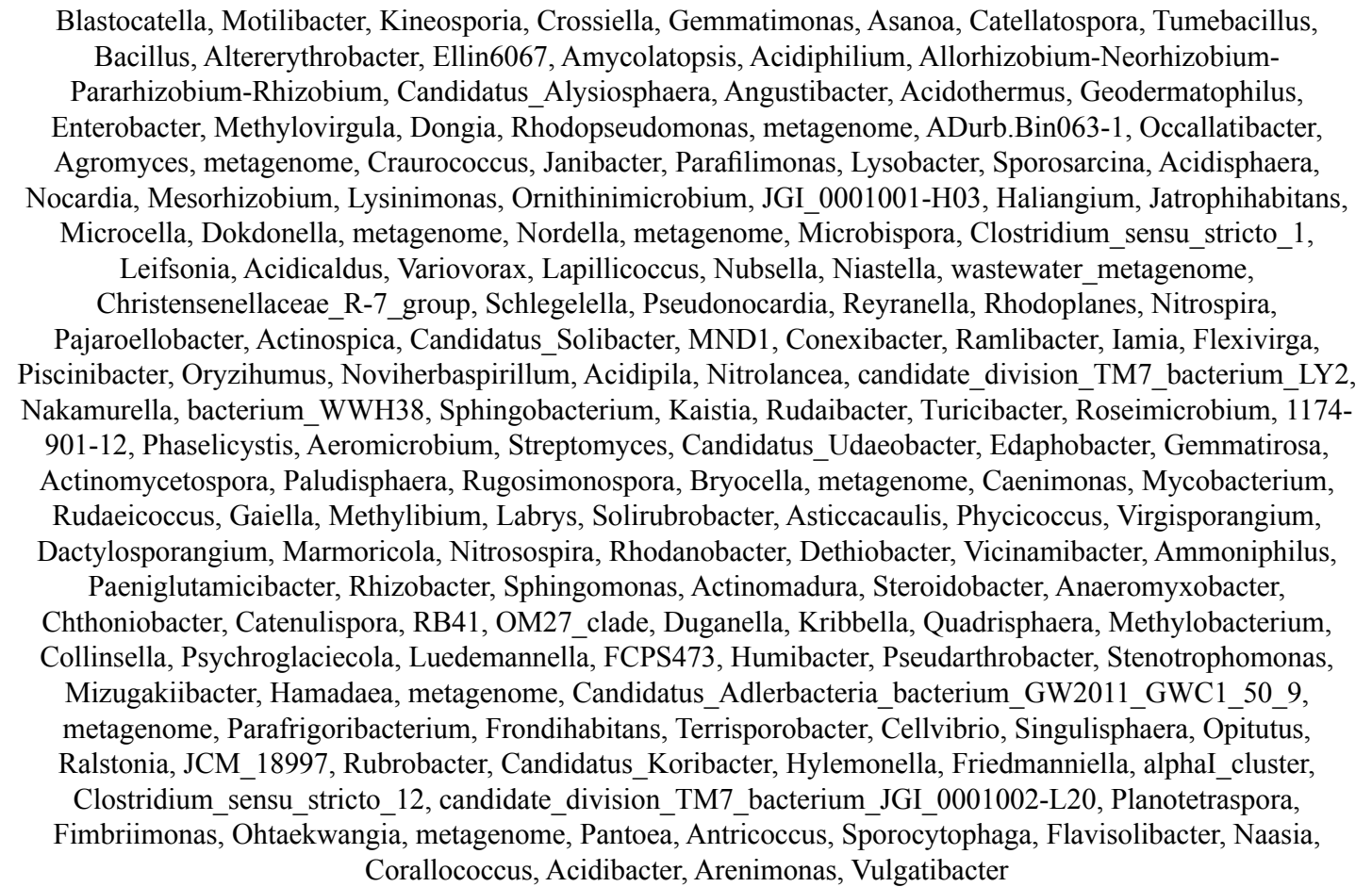 \\
\hline
\end{tabular}


Table S1. Continued.

\section{\begin{tabular}{|l|l}
\hline Virgibacillus, Rudaeicoccus, Flexivirga, Kineosporia, Ottowia, Humibacter, Acidicaldus, Dongia, \\
\end{tabular}}

Candidatus_Alysiosphaera, Bosea, Actinomycetospora, Conexibacter, Janibacter, Crossiella, Mycobacterium,

Rugosimonospora, Rhodopseudomonas, Haliangium, Pseudoclavibacter, Acidiphilium, Acidipila, Microcella, Angustibacter, Motilibacter, Dokdonella, Geodermatophilus, Rudaibacter, Methylibium, Bacillus, Parafilimonas, Mesorhizobium, Acidisphaera, Sporosarcina, Actinospica, Ellin6067, Sorangium, Gemmatimonas, Solirubrobacter, Phycicoccus, Oryzihumus, Sphingomonas, Cellvibrio, Antricoccus, Leifsonia, Georgenia, Marmoricola, Variovorax, Klugiella, Agromyces, Sphingobium, Actinoallomurus, Reyranella, Hylemonella, Jatrophihabitans, Acidothermus, Sinomonas, Qipengyuania, Dactylosporangium, Amycolatopsis, Candidatus Koribacter, Frigoribacterium, Devosia, FCPS473, metagenome, Nitrolancea, Segetibacter, Ralstonia, Bryocella, Methylocystis, Altererythrobacter, Longimycelium, OM27_clade, 1174-901-12, Tahibacter, Catellatospora, Pajaroellobacter, Methylovirgula, CL500-29_marine_group, Micrococcus, Quadrisphaera, Ramlibacter, JGI_0001001-H03, Tardiphaga, Kribbella, Ornithinimicrobium, Clostridium_sensu_stricto_1, Nitrospira, metagenome, Asticcacaulis, Aestuariimicrobium, Allorhizobium-Neorhizobium-Pararhizobium-Rhizobium, LD29, Knoellia, possible_genus_04, metagenome, Corallococcus, Collinsella, Streptomyces, Aeromicrobium, Sporichthya, Pedobacter, Pseudarthrobacter, Gemmatirosa, Ammoniphilus, Roseimicrobium, metagenome, bacterium_WWH38, metagenome, Rhodovastum, Chryseolinea, bacterium_Ellin6543, Kitasatospora, soil_ bacterium_WF55, Domibacillus, Nakamurella, Fodinibacter, Pantoea, JCM_18997, Candidatus_Solibacter, Streptosporangium, Labedaea, Leucobacter, Lysinibacillus, Labrys, Arthrobacter, Curtobacterium, Nocardia, Flavisolibacter, Pseudonocardia, Anaeromyxobacter, Dyadobacter, Vicinamibacter, Piscinibacter, Acinetobacter, wastewater_metagenome, Actinomadura, Vulgatibacter, Steroidobacter, Pseudomonas, Singulisphaera, Iamia,

Tumebacillus, Lysobacter, Dethiobacter, Paeniglutamicibacter, Kineosporia, Candidatus_Alysiosphaera,

Conexibacter, Craurococcus, Geodermatophilus, Quadrisphaera, Parafilimonas, Crossiella, Sporosarcina, Gemmatimonas, Naasia, Angustibacter, Mycobacterium, Janibacter, Rudaeicoccus, Mesorhizobium, Gemmatirosa, Anaeromyxobacter, Pseudonocardia, Dethiobacter, Flexivirga, Rhodopseudomonas, Humibacter, Lacunisphaera, Acidothermus, Modestobacter, Microbispora, Acidipila, Methylibium, Motilibacter, Subgroup_10, Nitrolancea, Bacillus, Blastococcus, Sphaerotilus, Sphingobium, Acidicaldus, Sphaerisporangium, Reyranella, Acidisphaera, Kitasatospora, Jatrophihabitans, Lysobacter, Ottowia, Chthonobacter, Fodinibacter, Streptomyces, Longimycelium, Luteolibacter, Cellvibrio, Polyangium, Ellin6067,

Mizugakiibacter, Flavisolibacter, Catenulispora, Ramlibacter, Knoellia, Sporichthya, Dokdonella, Amycolatopsis, Leifsonia, Acidiphilium, FCPS473, Microcella, JCM_18997, Christensenellaceae_R-7_group, Pseudarthrobacter, Oryzihumus, Chthonomonas, Parafrigoribacterium, Flavitalea, Phaselicystis, metagenome, Rhodanobacter, JGI_0001001-H03, Actinomycetospora, Haliangium, Steroidobacter, Phycicoccus, Solirubrobacter, Siphonobacter, Bryobacter, Rhodoplanes, Methylovirgula, Pajaroellobacter, Piscinibacter, Niastella, Vicinamibacter, Kribbella, Actinospica, Corallococcus, Tumebacillus, Clostridium_sensu_stricto_1, metagenome, Phytohabitans, Intrasporangium, Kaistia, Pelomonas, metagenome, Roseimicrobium, Gaiella, Altererythrobacter, Hamadaea, Catellatospora, Segetibacter, Luteibacter, Nonomuraea, Microvirga, Variovorax, Collinsella, Methylobacterium, Friedmanniella, Caenimonas, Marmoricola, Occallatibacter, Singulisphaera, Actinomadura, Candidatus_Udaeobacter, Acinetobacter, Rubrivivax, Romboutsia, Terrabacter, Arenimonas, Bradyrhizobium, Paludibaculum, metagenome, Lysinimonas, Pseudomonas, Methylocystis, 1174-901-12, Hylemonella, Brevundimonas, ADurb.Bin063-1, Iamia, Sphingomonas, Nitrospira, Agromyces, Rhizobacter, Turicibacter, wastewater_metagenome, Dongia, MND1, Actinomycetospora, Sphaerisporangium, Acidicaldus, Dongia, Amycolatopsis, Haliangium, Rhodopseudomonas, FCPS473, Geodermatophilus, Bacillus, Agromyces, MND1, Ramlibacter, Dactylosporangium, Acidothermus, Mycobacterium, Conexibacter, soil_bacterium_WF55, Dethiobacter, Angustibacter, Mesorhizobium, Gemmatimonas, Craurococcus, Kineosporia, Sphingomonas, Oryzihumus, Nitrolancea, Candidatus_Xiphinematobacter, Jatrophihabitans, Streptomyces, Catenulispora, Bauldia, Janibacter, Paralcaligenes, Nitrospira, Naasia, Acidibacter, Ellin6067, Reyranella, Actinospica, Knoellia, Microbispora, bacterium_WWH38, Acidisphaera, Humibacter, Rudaeicoccus, Solirubrobacter, Lysinimonas, Candidatus_Alysiosphaera, Friedmanniella, Gemmatirosa, Motilibacter, Opitutus, Saccharothrix, Methylibium, Methylovirgula, Ideonella, Lysinibacillus, Aquisphaera, Leifsonia, Nocardia, Roseimicrobium, Flavisolibacter, Phaselicystis, Chryseobacterium, Flexivirga, Parafilimonas, Piscinibacter, Sporichthya, Blastomonas, Acidiphilium, Crossiella, Nevskia, Sphingobium, JGI_0001001-H03, alphaI_cluster, Chitinophaga, 1174-90112, bacterium_Ellin6543, Chthonomonas, Pajaroellobacter, Candidatus_Adlerbacteria_bacterium_GW2011_ GWC1_50_9, Iamia, Marmoricola, Aeromicrobium, Qipengyuania, Nordella, Polycyclovorans, Lysobacter, Rhodanobacter, Luedemannella, Methylobacterium, Collinsella, Duganella, Acidipila, Pseudarthrobacter, Burkholderia-Caballeronia-Paraburkholderia, Pseudomonas, Siphonobacter, Rugosimonospora, Sinomonas, Phycicoccus, metagenome, Ottowia, Corallococcus, Rhodoplanes, Frondihabitans, wastewater_metagenome, Niastella, Pedobacter, Actinomadura, Kitasatospora, Gaiella, Noviherbaspirillum, metagenome, Acidovorax, Psychroglaciecola, metagenome, Asanoa, Sporosarcina, Microcella, Lapillicoccus, Schlegelella, Micromonospora, Variovorax, Sphingobacterium, Hylemonella, Candidatus_Udaeobacter, Altererythrobacter, Longimycelium, metagenome, metagenome, metagenome 
Table S1. Continued.

\begin{tabular}{|c|c|}
\hline BS90-SS90 & $\begin{array}{l}\text { Paeniglutamicibacter, Kineosporia, Candidatus_Alysiosphaera, Conexibacter, Craurococcus, Geodermatophilus, } \\
\text { Quadrisphaera, Parafilimonas, Crossiella, Sporosarcina, Gemmatimonas, Naasia, Angustibacter, Mycobacterium, } \\
\text { Janibacter, Rudaeicoccus, Mesorhizobium, Gemmatirosa, Anaeromyxobacter, Pseudonocardia, Dethiobacter, } \\
\text { Flexivirga, Rhodopseudomonas, Humibacter, Lacunisphaera, Acidothermus, Modestobacter, Microbispora, } \\
\text { Acidipila, Methylibium, Motilibacter, Subgroup_10, Nitrolancea, Bacillus, Blastococcus, Sphaerotilus, } \\
\text { Sphingobium, Acidicaldus, Sphaerisporangium, Reyranella, Acidisphaera, Kitasatospora, Jatrophihabitans, } \\
\text { Lysobacter, Ottowia, Chthonobacter, Fodinibacter, Streptomyces, Longimycelium, Luteolibacter, } \\
\text { Cellvibrio, Polyangium, Ellin6067, Mizugakiibacter, Flavisolibacter, Catenulispora, Ramlibacter, Knoellia, } \\
\text { Sporichthya, Dokdonella, Amycolatopsis, Leifsonia, Acidiphilium, FCPS473, Microcella, JCM_18997, } \\
\text { Christensenellaceae_R-7_group, Pseudarthrobacter, Oryzihumus, Chthonomonas, Parafrigoribacterium, Flavitalea, } \\
\text { Phaselicystis, metagenome, Rhodanobacter, JGI_0001001-H03, Actinomycetospora, Haliangium, Steroidobacter, } \\
\text { Phycicoccus, Solirubrobacter, Siphonobacter, Bryobacter, Rhodoplanes, Methylovirgula, Pajaroellobacter, } \\
\text { Piscinibacter, Niastella, Vicinamibacter, Kribbella, Actinospica, Corallococcus, Tumebacillus, Clostridium_sensu_ } \\
\text { stricto_1, metagenome, Phytohabitans, Intrasporangium, Kaistia, Pelomonas, metagenome, Roseimicrobium, } \\
\text { Gaiella, Altererythrobacter, Hamadaea, Catellatospora, Segetibacter, Luteibacter, Nonomuraea, Microvirga, } \\
\text { Variovorax, Collinsella, Methylobacterium, Friedmanniella, Caenimonas, Marmoricola, Occallatibacter, } \\
\text { Singulisphaera, Actinomadura, Candidatus_Udaeobacter, Acinetobacter, Rubrivivax, Romboutsia, Terrabacter, } \\
\text { Arenimonas, Bradyrhizobium, Paludibaculum, metagenome, Lysinimonas, Pseudomonas, Methylocystis, 1174-901- } \\
\text { 12, Hylemonella, Brevundimonas, ADurb.Bin063-1, Iamia, Sphingomonas, Nitrospira, Agromyces, Rhizobacter, } \\
\text { Turicibacter, wastewater_metagenome, Dongia, MND1 }\end{array}$ \\
\hline BS150-SS150 & $\begin{array}{l}\text { Actinomycetospora, Sphaerisporangium, Acidicaldus, Dongia, Amycolatopsis, Haliangium, Rhodopseudomonas, } \\
\text { FCPS473, Geodermatophilus, Bacillus, Agromyces, MND1, Ramlibacter, Dactylosporangium, Acidothermus, } \\
\text { Mycobacterium, Conexibacter, soil_bacterium_WF55, Dethiobacter, Angustibacter, Mesorhizobium, } \\
\text { Gemmatimonas, Craurococcus, Kineosporia, Sphingomonas, Oryzihumus, Nitrolancea, Candidatus_- } \\
\text { Xiphinematobacter, Jatrophihabitans, Streptomyces, Catenulispora, Bauldia, Janibacter, Paralcaligenes, } \\
\text { Nitrospira, Naasia, Acidibacter, Ellin6067, Reyranella, Actinospica, Knoellia, Microbispora, bacterium_- } \\
\text { WWH38, Acidisphaera, Humibacter, Rudaeicoccus, Solirubrobacter, Lysinimonas, Candidatus_Alysiosphaera, } \\
\text { Friedmanniella, Gemmatirosa, Motilibacter, Opitutus, Saccharothrix, Methylibium, Methylovirgula, } \\
\text { Ideonella, Lysinibacillus, Aquisphaera, Leifsonia, Nocardia, Roseimicrobium, Flavisolibacter, Phaselicystis, } \\
\text { Chryseobacterium, Flexivirga, Parafilimonas, Piscinibacter, Sporichthya, Blastomonas, Acidiphilium, } \\
\text { Crossiella, Nevskia, Sphingobium, JGI_0001001-H03, alphaI_cluster, Chitinophaga, 1174-901-12, } \\
\text { bacterium_Ellin6543, Chthonomonas, Pajaroellobacter, Candidatus_Adlerbacteria_bacterium_GW2011_- } \\
\text { GWC1_50_9, Iamia, Marmoricola, Aeromicrobium, Qipengyuania, Nordella, Polycyclovorans, Lysobacter, } \\
\text { Rhodanobacter, Luedemannella, Methylobacterium, Collinsella, Duganella, Acidipila, Pseudarthrobacter, } \\
\text { Burkholderia-Caballeronia-Paraburkholderia, Pseudomonas, Siphonobacter, Rugosimonospora, Sinomonas, } \\
\text { Phycicoccus, metagenome, Ottowia, Corallococcus, Rhodoplanes, Frondihabitans, wastewater_metagenome, } \\
\text { Niastella, Pedobacter, Actinomadura, Kitasatospora, Gaiella, Noviherbaspirillum, metagenome, Acidovorax, } \\
\text { Psychroglaciecola, metagenome, Asanoa, Sporosarcina, Microcella, Lapillicoccus, Schlegelella, Micromonospora, } \\
\text { Variovorax, Sphingobacterium, Hylemonella, Candidatus_Udaeobacter, Altererythrobacter, Longimycelium, } \\
\text { metagenome, metagenome, metagenome, }\end{array}$ \\
\hline
\end{tabular}

